\title{
Incidencia de la hemorragia intraventricular en prematuros de muy bajo peso y sus factores asociados en un hospital nacional de Lima, Perú
}

Incidence of intraventricular hemorrhage in very low birth weight preterm infants and associated factors at a national hospital in Lima, Peru

Olga Lizama ${ }^{1, a, c}$, Herminio Hernández ${ }^{2, b}$, Fabiola Rivera ${ }^{2, b, c}$, Alfredo Tori $^{1, b, d}$

\section{RESUMEN}

Objetivos: Determinar la incidencia de hemorragia intraventricular (HIV) en un hospital nacional y los factores asociados. Material y métodos: Estudio Caso-control, retrospectivo, en una cohorte anidada desde julio 2010 hasta abril 2012. El diagnóstico se realizó por ecografía cerebral. Resultado: La incidencia anual general fue $33,53 \%$. En las primeras 72 horas, la incidencia anual fue 17,23\% y el factor asociado fue la edad gestacional $\leq$ 28 semanas (OR: 2,41, IC: 1,10-5,34). La incidencia anual de HIV después de las 72 h hasta el séptimo día fue $16,30 \%$ y los factores asociados fueron: las complicaciones del soporte ventilatorio, como hipercapnia, neumotórax, hipocapnia y atelectasia (OR: 4,33, IC: 1,26-14,81). Conclusión: La incidencia anual general fue alta, y el factor asociado encontrado en las primeras 72 horas fue la menor edad gestacional y después de las 72 horas fueron las complicaciones del soporte ventilatorio.

PALABRAS CLAVE: Incidencia, recién nacido de muy bajo peso, hemorragia intracraneal, hemorragia cerebral. (Fuente: DeCS BIREME).

\section{SUMMARY}

Objectives: Determine the incidence of intraventricular hemorrhage ( $\mathrm{IH})$ in a national hospital and its associated factors. Methods: Nested case-control study conducted from July 2010 to April 2012. Diagnosis was confirmed by cerebral ultrasound. Results: Overall annual incidence was $33.53 \%$. During the first 72 hours, the annual incidence was $17.23 \%$, and the associated factor was gestational age $\leq 28$ weeks (OR: 2.41, IC: 1.10-5.34). Annual incidence of IH from 72 hours to 7 days was $16.30 \%$, and associated factors were ventilatory support complications such as hypercapnia, pneumothorax, hypocapnia and atelectasis (OR: 4.33, IC: 1.26-14.81). Conclusion: The overall annual incidence of IH was high. Low gestational age was associated with IH in the first 72 hours, while complications of ventilatory support were associated with IH after 72 hours.

KEYWORDS: Incidence, infant, very low birth weight, intracranial hemorrhages, cerebral hemorrhage (Source: MeSH NLM)

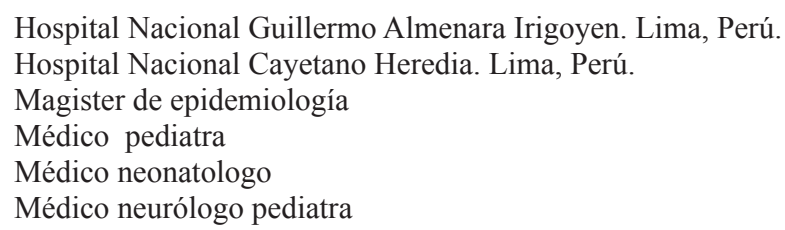




\section{INTRODUCCIÓN}

La hemorragia intraventricular es la forma más frecuente de hemorragia intracraneal neonatal, y es característica del prematuro, iniciándose típicamente en la matriz germinal, que es una zona conformada por células precursoras de la glía ricamente vascularizada. Su etiología es multifactorial, atribuido principalmente a la fragilidad de los vasos de la matriz germinal y disturbios en el flujo cerebral. La importancia de esta lesión no solo se relaciona con su incidencia y mortalidad significativa, sino también con las secuelas como parálisis cerebral, retardo mental, y crisis epilépticas en los neonatos afectados (1-5), las cuales son generalmente directamente proporcionales al grado de severidad de la hemorragia intraventricular.

En Estados Unidos cerca de 12 mil prematuros presentan hemorragia intraventricular cada año. La incidencia ha disminuido a través del tiempo, en los inicios de los ochenta principalmente en los nacidos prematuros con muy bajo peso al nacer $(<1500 \mathrm{~g})$ era 40 a $50 \%$, y llegó a disminuir hasta $20 \%$ a finales de los ochenta (1-7). Esta incidencia se ha mantenido constante en las últimas dos décadas porque los límites de viabilidad se han extendido hacia las 23 a 25 semanas de edad gestacional $(6,7)$.

En nuestro medio existen pocos reportes, Guillén (8) en su estudio de cohorte prospectiva en el Hospital Nacional Cayetano Heredia, en neonatos con menos de 34 semanas de edad gestacional, encontró una incidencia de 44,4\%. Un Jan (9), reportó una incidencia de $29,7 \%$ en neonatos con peso entre 500 y $1499 \mathrm{~g}$ en el Hospital Guillermo Almenara Irigoyen y Castañeda (10), en $30,8 \%$ en una serie de casos retrospectiva de neonatos prematuros con peso menor de $1500 \mathrm{~g}$ y menos de 36 semanas, en el mismo hospital. Medina (11) en un estudio retrospectivo en el Hospital Nacional Cayetano Heredia, encontró una incidencia de 34,7\% en prematuros de menos de 34 semanas, observándose una tendencia a disminuir a través del tiempo, aunque mayor que lo reportado en la literatura mundial y de ahí la importancia de identificar los factores asociados.

Estos factores asociados serian aquellos que estarían relacionados con la alteración del flujo sanguíneo cerebral o elevación de la presión venosa cerebral, como son los problemas de oxigenación secundarios a la dificultad respiratoria: enfermedad de membrana hialina que muchas veces requiere soporte ventilatorio y las complicaciones de dicho soporte como son: la presencia de neumotórax, acidosis y atelectasias (12-14), además de otros factores que complican o acompañan la depresión neonatal, como: la sepsis, la necesidad de uso de medicamentos (cristaloides e inotrópicos) y componentes sanguíneos en bolo, así como la alteración en el perfil de coagulación (1$3,12,13,15,16)$.

Además, la hemorragia intraventricular, patología frecuente en los prematuros de muy bajo peso se diagnóstica mediante la ecografía cerebral, que es un método relativamente sencillo, no invasivo y económico pero depende de la experiencia del examinador; según reportes de la literatura a las 24 horas de nacido, se pueden identificar el $50 \%$ de los casos de hemorragias intraventriculares, a las 72 horas se identificaran el $90 \%$ y a los 7 días alrededor del $99 \%$ de los casos $(2,3,6,17,18)$.

Por lo mencionado el objetivo de este estudio fue determinar la incidencia de hemorragia intraventricular en prematuros de muy bajo peso y los factores asociados, en un hospital de nivel IV de EsSALUD.

\section{MATERIAL Y METÓDOS}

\section{Tipo y lugar de estudio}

Estudio observacional, retrospectivo, realizado en el servicio de Neonatología del Hospital Guillermo Almenara Irigoyen de Lima, Perú. Los casos incidentes se recolectaron de la cohorte de nacimientos de recién nacidos prematuros, desde julio de 2010 hasta abril de 2012. Para determinar los factores asociados, se utilizó el estudio de casos y controles anidado en una cohorte, en dos momentos: Grupo I: $\leq 72$ horas y Grupo II: > 72 horas $\mathrm{y} \leq 7$ días.

\section{Población y tamaño de la muestra}

Se calculó el tamaño de una muestra representativa para la incidencia anual en nuestro hospital, con el Programa EpiInfo v. 6, con IC del 99\%, con una frecuencia esperada de $40 \%$ y un peor valor de $35 \%$, resultando en 75 neonatos, El tamaño de la muestra para los factores asociados, en el Grupo I fue de 36 prematuros de muy bajo peso para los casos y 72 para los controles (Relación 1:2); en el Grupo II fue 30 prematuros de muy bajo peso para los casos y 90 para los controles (Relación 1:3), basándose en la asunción de un error tipo I de $0,05 \mathrm{y}$ un poder de $80 \%$.

\section{Criterios de inclusión}

Casos: Prematuros de $<1500 \mathrm{~g}$ y con edad gestacional $\leq 32$ semanas, con $>24$ horas de vida 
y nacidos en el Hospital Guillermo Almenara Irigoyen, con diagnóstico ecográfico de hemorragia intraventricular, según los criterios de Volpe $(1,2,5)$.

Controles: Prematuros de $<1500$ g y con edad gestacional $\leq 32$ semanas con $>24$ horas de vida $\mathrm{y}$ nacidos en el Hospital Guillermo Almenara Irigoyen, sin hemorragia intraventricular en la ecografía cerebral.

\section{Criterios de exclusión}

Presencia de malformaciones genéticas mayores y síndromes dismorficos con compromiso cerebral.

\section{Definiciones operacionales}

Hemorragia intraventricular: Variable dependiente, que se define como la presencia de sangre en el espacio subependimario de la matriz germinal, diagnóstico realizado por ecografía. Los grados de severidad se determinaron por los criterios propuestos por Volpe en 2001, utilizando el ecógrafo. Grado I: Limitado a la matriz germinal $(<10 \%)$, Grado II: Compromete entre el 10 al 50\% del ventrículo lateral. Grado III: Cuando invade más del $50 \%$ del ventrículo lateral, acompañado de dilatación ventricular. Entidad aparte (equivale al grado IV de Papile) Compromiso parenquimal $(1,2,5,6)$.

Edad gestacional: Se categorizó en $\leq 28$ semanas y $>28$ y $\leq 32$ semanas $(15,19)$.

Peso: Se categorizó en $<1000 \mathrm{~g} \mathrm{y} \geq 1000 \mathrm{y}<1500$ g (15).

Sexo: Se categorizó en hombre o mujer (19).

Depresión neonatal: Se categorizó en presencia de depresión neonatal con Apgar $\leq 3$ al minuto o a los cinco minutos o ausencia $(12,13)$.

Uso de ventilación a presión positiva al nacer con o sin intubación: Se categorizó en: si fue requerida la ventilación a presión positiva o no fue requerida (12).

Enfermedad de membrana hialina: Se categorizó en presencia o ausencia.

Necesidad de soporte ventilatorio: Se categorizó en necesitó o ventilación mecánica o uso presión positiva continua en la vía aérea o no necesitó (14).
Complicaciones del soporte ventilatorio: Se categorizó en presencia de una o más complicaciones o no complicaciones. Las complicaciones consideradas fueron: Neumotórax evidenciado en la radiografía de tórax (13), Hipercapnia: $\mathrm{PCO}_{2}>55$, Hipocapnia: $\mathrm{PCO}_{2}<35$ y Atelectasia: imagen radiopaca de la zona afectada evidenciada en la radiografía de tórax. $(14,18,20,21,22,23)$.

Sepsis Neonatal: Se categorizó en si estaba presente o no.

Necesidad de infusiones en bolos de bicarbonato, componentes sanguíneos, cloruro de sodio o inotrópicos: se categorizó en sí o no.

Alteración del perfil de coagulación: Se categorizó en si estuvo presente o no $(1,24,25)$.

Hemorragia intraventricular severa: Según los criterios de Volpe, el grado 3 y el compromiso parenquimal. Se categorizó en sí o no.

\section{Procedimiento}

El tipo de muestreo fue no probabilístico, consecutivo y por conveniencia desde julio del 2010 hasta abril del2012, basándose en el libro de nacimientos y completándose los datos con sus historias clínicas, utilizándose una ficha para la recolección de datos. El diagnóstico de la hemorragia intraventricular fue realizado por los médicos del Servicio de Radiología, utilizando un ecógrafo portátil, marca Aloka modelo SSD-30, con un transductor lineal de 7,545 MHz.

\section{Consideraciones éticas}

El protocolo fue aprobado por el Comité de Ética de investigación del Hospital Guillermo Almenara Irigoyen y de la Universidad Peruana Cayetano Heredia. El estudio fue realizado siguiendo los principios de la declaración de Helsinki.

\section{Análisis estadístico}

Se utilizó estadística descriptiva, se determinaron porcentajes en las variables nominales y medidas de tendencia central y desviación estándar en las numéricas en cada uno de los grupos mencionados anteriormente. En la parte analítica a las variables categóricas se les realizó un análisis bivariado utilizando tablas de contingencia determinando la medida de asociación en términos de OR, con un IC 95\% y posteriormente a las 


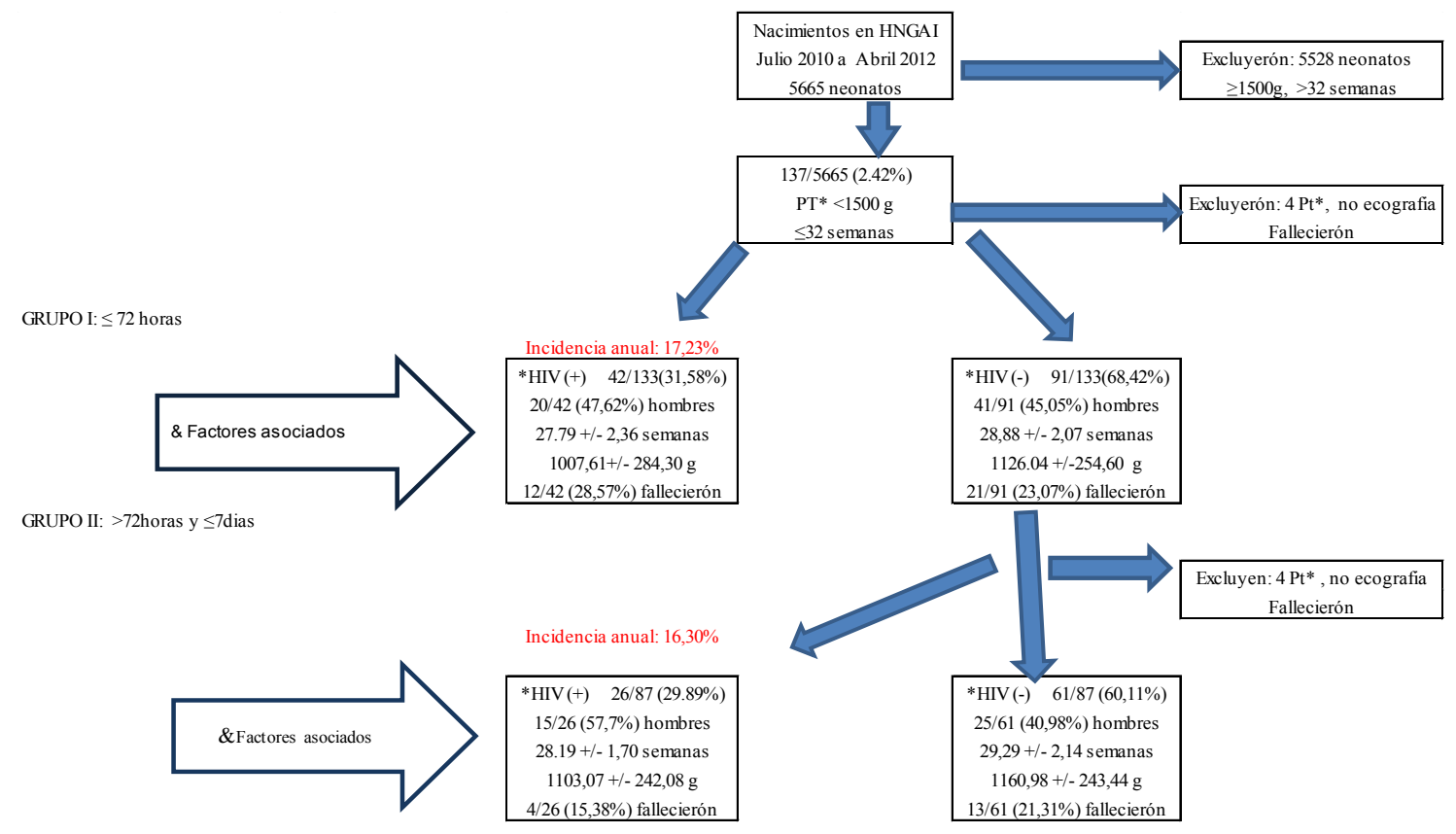

\section{*HIV: Hemorragia intraventricular, PT: Neonato Pretermino}

\& Factores asociados: Edad gestacional $<28$ sem, Peso nacer $<1000 \mathrm{~g}$, sexo masculino, depresión al nacer (Apgar $\leq$

3) al minuto y 5 minutos, VPP al nacer, bolos endovenoso, perfil de coagulación

Gráfico 1. Frecuencias de presentación de hemorragia intraventricular en el Hospital Nacional Guillermo Almenara Irigoyen en el período de estudio (22 meses).

asociaciones que tenían significancia estadística se les realizo un análisis regresión logística, para determinar si realmente estaban asociadas con la presencia de hemorragia intraventricular y no eran variables confusoras.

\section{RESULTADOS}

En el período del estudio, julio del 2010 hasta abril del 2012, nacieron 5665 prematuros en el Hospital Almenara Irigoyen, 137 (2,42\%) fueron menores de 1500 g y tenían $\leq 32$ semanas al nacer. De ellos a 4 prematuros no se pudo realizar la primera ecografía cerebral porque fallecieron antes del procedimiento (Grupo I $\leq 72$ horas) y 4 fallecieron antes de realizarse la segunda ecografía cerebral (Grupo II $>72$ horas y $\leq 7$ días), solo se les pudo hacer la primera ecografía cerebral (Gráfico 1).

Laincidencia general de hemorragia intraventricular en los prematuros de muy bajo peso que fue $33,53 \%$ por año (Gráfico 1). La incidencia en el grupo I fue $17,23 \%$ por año y en el grupo II fue $16,30 \%$ por año.
La distribución de los pacientes con hemorragia intraventricular según el grado, se pueden apreciar en la tabla 1, se observa que en el grupo I, el grado II fue el más frecuente y en el grupo II, el grado I fue el más frecuente.

La edad gestacional de los prematuros con hemorragia intraventricular en el grupo I fue 27,80 \pm 2,40 semanas y en el grupo II fue 28,20 $\pm 1,70$ semanas.

Tabla 1. Severidad de la Hemorragia Intraventricular.

\begin{tabular}{lccccc}
\hline & Grupo I & \multicolumn{2}{c}{ Grupo II } \\
\hline & n & $\%$ & \multicolumn{1}{c}{ n } & $\%$ \\
Grado I & 10 & 23,7 & 13 & 50,0 \\
Grado II & 24 & 57,1 & 12 & 46,2 \\
Grado III & 4 & 9,6 & 0 & 0,0 \\
Compromiso parenquimal & 4 & 9,6 & 1 & 3,8 \\
TOTAL & 42 & & 26 & \\
\hline
\end{tabular}

Grupo I: $\leq 72$ horas; Grupo II: $>72$ horas $\mathrm{y} \leq 7$ días 
Tabla 2. Factores asociados. Modelos crudos.

\begin{tabular}{lcccccc}
\hline & \multicolumn{3}{c}{ Grupo I } & & \multicolumn{3}{c}{ Grupo II } \\
& OR & IC 95\% & p & OR & IC 95\% & p \\
\hline Edad gestacional $\leq 28$ semanas & 2,91 & $1,27-6,81$ & $<0,05$ & 3,25 & $1,13-9,45$ & $<0,05$ \\
Peso al nacer $<1000 \mathrm{~g}$ & 2,18 & $0,94-4,99$ & $<0,05$ & 1,49 & $0,46-4,61$ & $>0,05$ \\
Sexo masculino & 1,11 & $0,50-2,46$ & $>0,05$ & 1,96 & $0,70-5,55$ & $>0,05$ \\
Depresión neonatal (APGAR $\leq 3$ al minuto)* & 1,41 & $0,33-5,23$ & $>0,05$ & 4,61 & $0,79-31,58$ & $<0,05$ \\
Ventilación a presión positiva & 2,58 & $1,14-5,85$ & $<0,05$ & 2,81 & $0,97-8,13$ & $<0,05$ \\
Enfermedad Membrana hialina & 1,71 & $0,68-4,60$ & $>0,05$ & 3,82 & $1,10-16,88$ & $<0,05$ \\
Soporte ventilatorio & 1,48 & $0,50-4,93$ & $>0,05$ & 1,76 & $0,38-11,23$ & $>0,05$ \\
Complicaciones del soporte ventilatorio & 1,28 & $0,53-3,10$ & $>0,05$ & 5,41 & $1,45-22,29$ & $<0,05$ \\
Bolos endovenosos** & 1,66 & $0,62-4,31$ & $>0,05$ & 2,84 & $0,73-10,78$ & $>0,05$ \\
Perfil de coagulación alteraado & 2,08 & $0,78-5,44$ & $>0,05$ & 2,25 & $0,48-9,89$ & $>0,05$ \\
Sepsis neonatal & 0,42 & $0,08-3,95$ & $>0,05$ & 3,84 & $0,41-47,99$ & $>0,05$ \\
\hline
\end{tabular}

* No hay neonatos con Apgar $\leq 3$

** Cloruro de sodio, componentes sanguíneos

Grupo I: $\leq 72$ horas; Grupo II: $>72$ horas $\mathrm{y} \leq 7$ días

Tabla 3. Factores asociados. Modelo ajustado.

\begin{tabular}{lccc}
\hline & OR & IC 95\% & p \\
\hline Grupo I & & & \\
$\quad$ Edad gestacional $\leq 28$ semanas & 2,40 & $1,10-5,34$ & $<0,05$ \\
$\quad$ Ventilación a presión positiva al nacer & 2,03 & $0,92-4,45$ & $>0,05$
\end{tabular}

\section{Grupo II}

\begin{tabular}{lccc} 
Edad gestacional $\leq 28$ semanas & 2,14 & $0,65-7,03$ & $>0,05$ \\
Complicaciones en soporte ventilatorio & 4,33 & $1,26-14,80$ & $<0,05$ \\
Enfermedad Membrana hialina & 1,87 & $0,33-12,36$ & $>0,05$ \\
\hline
\end{tabular}

Grupo I: $\leq 72$ horas; Grupo II: $>72$ horas $\mathrm{y} \leq 7$ días

El peso al nacer fue $1007,61 \pm 284,30 \mathrm{~g}$ en el grupo I y $1103,07 \pm 242,08 \mathrm{~g}$ en el grupo II en los prematuros que presentaron hemorragia intraventricular. No se encontró diferencias respecto al sexo en ambos grupos. La mortalidad asociado a hemorragia intraventricular en el grupo I fue $28,57 \%$ y en el grupo II $15,38 \%$ no observándose significancia estadística (Gráfico 1).
Para evaluar los factores asociados: en el grupo I se compararon 42 casos con 91 controles y para el grupo II se comparó 26 casos y 81 controles. El OR en el grupo I fue significativo $(p<0,05)$ para los factores edad gestacional $\leq 28$ semanas y ventilación a presión positiva al nacer (Tabla 2). El análisis de regresión logística para eliminar variables de confusión mostró 
que tener $\leq 28$ semanas al nacer se asoció en 2.41 veces más probabilidad de tener hemorragia intraventricular (IC: 1,10-5,34). No se consideró el peso $<1000 \mathrm{~g}$ porque el intervalo de confianza incluía a la unidad (Tabla 3).

El OR en el grupo II fue significativo $(\mathrm{p}<0,05)$ para los factores edad gestacional $\leq 28$ semanas (IC: 1,13-9,45), enfermedad de membrana hialina (IC: $1,1-16,88)$ y complicaciones del soporte ventilatorio (IC: 1,45-22,29) (Tabla 1). No se consideraron las variables depresión neonatal al minuto (IC: 0,79$31,58)$ y ventilación a presión positiva (IC: $0,97-8,13$ ) porque el intervalo de confianza incluyó a la unidad (Tabla 2). El análisis de regresión logística mostró que tener complicaciones del soporte ventilatorio como hipercapnia, neumotórax, hipocapnea y atelectasia, se asociaba en 4,33 veces más probabilidad de tener hemorragia intraventricular (IC: 1,26-14,8) (Tabla 3).

\section{DISCUSIÓN}

En nuestro estudio se observó una incidencia general anual de HIV en prematuros de $\leq 32$ semanas con muy bajo peso al nacer de $33,53 \%$, siendo alta comparado con lo reportado, que desde los ' 80 es alrededor del $25 \%(1,2,7,16)$. El 17,23\% ocurrió en las primeras 72 horas y $16,30 \%$ después de las 72 horas; llama aún más la atención la alta incidencia después de las 72 horas, dado que en la literatura se reporta que debería ser alrededor del 10\% más de lo que se presentó en las primeras 72 horas; esto podría deberse al tipo de atención dado en nuestra unidad de cuidados intensivos, además de las características de nuestra población.

Por esto debemos mencionar entre los sesgos el de membrecía o selección de la población de estudio, ya que está constituida por prematuros de alto riesgo, cuyas madres han sido gestantes con patología compleja referidas de diversos puntos del país pertenecientes a la Red del Hospital Guillermo Almenara Irigoyen. Además esta población fue seleccionada por un muestro de tipo no probabilístico por conveniencia, lo cual es un riesgo importante de representatividad.

También debemos mencionar el sesgo de medición porque influyó la experiencia de los médicos radiólogos que realizaron la ecografía cerebral, en una sola oportunidad, y no se realizó un estudio de concordancia entre los radiólogos, por razones de tipo logístico (elevado número de pacientes citados). Otro sesgo importante fue el uso de un transductor lineal que no sería el ideal para hacer el diagnóstico de esta patología, debido a que tiene un punto de base más pequeño que el transductor convexo el que permite una mejor resolución para estructuras cercanas, aunque para estructuras más alejadas ambos tienen una resolución limitada aunque mayor en el transductor lineal porque el haz de ultrasonido es divergente (26).

Un punto importante es que la frecuencia de las formas severas (grado III y compromiso parenquimal: $19,2 \%$ en el grupo I y $3,85 \%$ en el grupo II), fue menor al reportado en otros estudios, que reportan en alrededor de 35\%. Estas serían las que presentarían con mayor frecuencia secuelas neurológicas, siendo un punto a favor en el pronóstico neurológico de nuestros prematuros, a pesar del alta incidencia (2).

En cuanto a los factores asociados, en el grupo I, el tener $\leq 28$ semanas al nacer tenía 2,41 veces más probabilidad de presentar hemorragia intraventricular; esto ha sido reportado en múltiples estudios $(1,2,7-$ 10), y se explicaría por la inmadurez de la matriz germinal que se encuentra altamente vascularizada y podría sangrar en cualquier momento.

En el grupo II, el único factor asociado significativo fue presentar complicaciones en el soporte ventilatorio y que incrementaba en 4,33 veces la probabilidad de desarrollar la hemorragia intraventricular. Las complicaciones encontrada fueron: acidosis respiratoria $(40 \%)$ y neumotórax $(33,3 \%)$ principalmente, que estarían relacionados con una alteración del flujo cerebral y como consecuencia el desarrollo de esta patología $(15,13,16,18,14,21,22,23)$. Estos hallazgos son importantes porque hacen necesario capacitar al personal médico y de enfermería en las nuevas terapias ventilatorias y seguimiento cercano de nuestros pacientes, y disponer de monitores apropiados.

Una limitación importante del estudio fue que no se llegó a completar el tamaño de la muestra del grupo II, lo cual ampliaría el intervalo de confianza, es decir la posibilidad que los datos hallados no sean exactos en este grupo.

Concluimos que la incidencia fue alta, probablemente debido al muestro no probabilístico utilizado y los otros sesgos mencionados. Se recomienda realizar estudios multicéntricos de casos y controles para poder completar el tamaño de muestra para determinar los factores asociados, dado que nuestro estudio en este aspecto fue solo exploratorio. 


\section{Declaración de Financiamiento y de conflictos de interés:}

Los autores declaramos que no se recibió apoyo económico o de cualquier otra índole de alguna institución o persona en particular. Asimismo, declaramos no tener conflictos de intereses.

\section{Contribución de autoría:}

OL participó en el diseño del estudio, análisis e interpretación de los datos, redacción del artículo y aprobación de la versión final. HH, FR y AT, participaron en el diseño del estudio, análisis e interpretación de los datos, revisión crítica del artículo y aprobación de la versión final.

\section{Correspondencia:}

Dra. Olga Cecilia Lizama Olaya

Av. Angélica Gamarra 368. Urb. El Trébol. Los

Olivos

Olgaliza@hotmail.com

\section{REFERENCIAS BIBLIOGRÁFICAS}

1. Ballabh P. Intraventricular hemorrhage in premature infants: mechanism of disease. Pediatr Res. 2010; 67(1):1-18

2. Whitelaw A. Core Concepts: intraventricular hemorrhage. Neo Reviews. 2011; 12: c94-c101.

3. McCrea H, Ment L. The diagnosis, management and postnatal prevention of intraventricular hemorrhage in the preterm neonate. Clin Perinatol. 2008; 35(4): $1-17$.

4. Ment LR, Vorh B. Change in cognitive function over time in very low-birth-weigth infants. JAMA. 2003; 289:705-711.

5. Madan A, Hamrick S, Ferreiro D. Central nervous system and neuroprotection. En: Taeusch W, Ballard R, Gleason C. Avery's Diseases of the Newborn. 8th Edition. Philadelphia: Elseviers Saunders; 2005. p. 965-969.

6. Gonzalez H. Hemorragia intracraneana. En: Manual de Neonatología. Segunda Edición. Santiago de Chile: Publicaciones Técnicas Mediterráneo; 2001.p. 379-384.

7. Sheth R. Trends in incidence and severity of intraventricular hemorrhage. J Child Neuro. 1998; 13: 261-264.

8. Guillén D. Diagnóstico de las lesiones cerebrales en los prematuros menores de 34 semanas: incidencia, factores asociados y pronóstico. Tesis para optar el grado de doctor en medicina. Lima, Perú. Universidad Peruana Cayetano Heredia, 2004. 100 pp.

9. Un Jan R, Hernández H, Loza C, Huayanay L. Sobrevida del recién nacido menor de $1500 \mathrm{~g}$ y factores que lo afectan en el servicio de Neonatología del Hospital Nacional Guillermo Almenara- Años 2000 a 2002. Rev Per Ped. 2005; 58: 14-23.

10. Castañeda A. Incidencia y factores asociados a la hemorragia intraventricular en prematuros menores de $1500 \mathrm{~g}$. Servicio de neonatología. Hospital Nacional Guillermo Almenara I. Años 2000 a 2002. Tesis de magister en medicina. Lima, Perú. Universidad Peruana Cayetano Heredia, 2007. 49 pp.

11. Medina P, Rivera F, Tori A. Frecuencias, características y mortalidad asociadas a la enfermedad neurológica en la Unidad de Cuidados Intensivos Neonatales del Hospital Nacional Cayetano Heredia. Rev Per Ped. 2007; 60 (1): 11-19.

12. Segovia O. Hemorragia intraventricular en niños pretérmino, incidencia y factores de riesgo. Un estudio de corte transversal. Med UNAD. 2003; 6(17): 57-62.

13. Kadri $\mathrm{H}$. The incidence, timing, and predisposing factors of germinal matrix and intraventricular hemorrhage $(\mathrm{GMH} / \mathrm{IVH})$ in preterm neonates. Childs Nery Syst. 2006, 22: 1086-1090.

14. Mehrabani D. Association of pneumothorax and hypotension with intraventricular haemorrhage. Arch Dis Child. 1991; 66: 48-51.

15. Caro M. Evaluación de factores prenatales, perinatales y postnatales para hemorragia intraventricular en prematuros menores de 33 semanas de edad gestacional. Gac Med Mex. 2010; 146:376-382.

16. Khosravi N. Risk factors for intraventricular hemorrhage in very low birth weight infants. Iran J Ped. 2007; 17(2): 101-107.

17. Benavides V, Díaz H, Rodríguez N. Ultrasonografía de la Hemorragia intraventricular en Recién Nacidos Prematuros: factores de riesgo. Rev Per Radiol. 2005; 5(14): 20-26.

18. Soul JS. Intracranial hemorrhage. En: Coherty J, Stark A. Manual of Neonatal Care. Fifth Edition. Philadelphia: Lippincott Williams \& Wilkin; 2004.p. 527-535

19. Mohamed A. Male gender is associated with intraventricular hemorrhage. Pediatrics. 2010; 125: e333-e339.

20. Han-Suk K. Risk factor for periventricularIntraventricular hemorrhage in premature Infants. J Korean Med. 2010; 25:418-425.

21. Kaiser Jr. Hypercapnia during the first 3 days of life is associated with severe intraventricular hemorrhage in very low birth weight infants. J Perinatol. 2006; 6:279-285.

22. Fabres J. Both extremes of arterial carbon dioxide pressure and the magnitude of fluctuations in arterial carbon dioxide are associated with severe intraventricular Hemorrhage in preterm infants. Pediatrics. 2007; 119:299-305.

23. Levene MI. Risk factors in the development of intraventricular haemorrhage in the preterm neonate. Arch Dis Child. 1982; 57:410-417.

24. Beverley D. Intraventricular haemorrhage and haemostasis defects. Arch Dis Child. 1984; 59:444- 
448

25. Gooring A, Neufeld E. Bleeding. En: Coherty J, Stark A. Manual of Neonatal Care. Fifth Edition. Philadelphia: Lippincott Williams \& Wilkins. 2004.p. 468-474.

26. Leiser L, Steggerda S. Cranial Ultrasonagraphy In
Neonates: Roles and Limitations. Semin Perinatol. 2010; 34(1): 28-38.

Recibido: 20/11/2013

Aceptado: 20/05/2014 